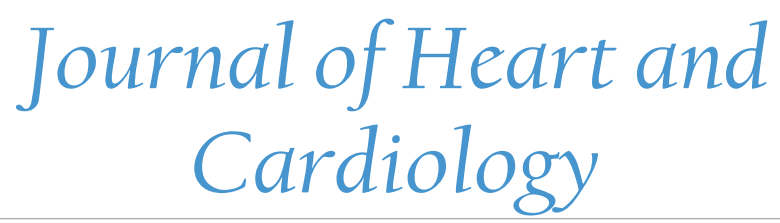

\title{
Upper Extremity Function after Transradial Percutaneous Coronary Intervention Short-Term Interim Results of the ARCUS Study
}

\author{
Alexander JJ Ijsselmuiden ${ }^{* \#}$, Carlo. A.J Holtzer ${ }^{2 \#, ~ E v a ~ M ~ Z w a a n ~}{ }^{3}$, Robert-Jan M van Geuns ${ }^{4}$, Niels \\ van Royen ${ }^{5}$, Esther Moerman ${ }^{6}$, Giovanni Amoroso ${ }^{7}$, Marco J.PF. Ritt ${ }^{8}$, Ton A Schreuders ${ }^{9}$, Marcel J.M \\ Kofflard $^{3}$
}

${ }^{1}$ Department of Cardiology, Amphia Hospital, Breda, the Netherlands

${ }^{2}$ Department of Plastic, Reconstructive, and Hand Surgery, Albert Schweitzer Hospital, Dordrecht, the Netherlands

${ }^{3}$ Department of Cardiology, Albert Schweitzer Hospital, Dordrecht, the Netherlands

${ }^{4}$ Department of Cardiology, Erasmus Medical Centre, Rotterdam, the Netherlands

${ }^{5}$ Department of Cardiology, VU University Medical Centre, Amsterdam, the Netherlands

${ }^{6}$ Department of Plastic, Reconstructive, and Hand Surgery, Onze Lieve Vrouwe Gasthuis, Amsterdam, the Netherlands

${ }^{7}$ Department of Cardiology, Onze Lieve Vrouwe Gasthuis, Amsterdam, the Netherlands

${ }^{8}$ Department of Plastic, Reconstructive, and Hand Surgery, VU University Medical Centre, Amsterdam, the Netherlands

${ }^{9}$ Hand and Pols centre, Dordrecht, the Netherlands

\#Authors contributed equally

*Corresponding author: IJsselmuiden, A.J.J., MD, PhD, Department of Cardiology, Amphia Hospital, Mailbox 901584800

RK Breda, The Netherlands, Tel: 0031-0765954090; Fax: 0031-076-5953340; E-mail id: sijsselmuiden@amphia.nl

\begin{abstract}
Aims: To examine the short-term effects of transradial percutaneous coronary interventions on the upper extremities function.

Method and results: This is an interim analysis of the Effects of trAnsRadial perCUtaneouS coronary intervention on upper extremity function (ARCUS) study. Out of 191 patients evaluated at 2 weeks after they had undergone a transradial percutaneous coronary intervention, $120(62.8 \%)$ had manifestations of upper extremity dysfunction on the side of the intervention. The main abnormalities were a decrease in sensibility, $a \geq 15 \%$ increase in the Disabilities of the Hand and Shoulder questionnaire score and $\mathrm{a} \geq 2 \mathrm{~cm}$ increase in hand and forearm volumes. Radial artery occlusions occurred in 12 patients in the upper extremity dysfunction versus 1 patient in the no upper extremity dysfunction group $(\mathrm{p}=0.03)$. Patients with upper extremity dysfunction were significantly more likely to have a family history of heart disease $(50 \%$ vs. $26.8 \% ; p=0.002)$. In addition, there was a trend $(p=0.07)$ toward a greater proportion of previous smokers in the group with upper extremity dysfunction than in the other group.

Conclusions: Upper extremity dysfunction after transradial percutaneous coronary intervention is a medical concern. However, our original score may be overly sensitive and overestimate the rate of upper extremity dysfunction. Further analyses are needed, as well as perhaps a modification of the primary endpoint.
\end{abstract}

Received Date: May 18, 2017

Accepted Date: June 28, 2017

Published Date: July 06, 2017

Citation: IJsselmuiden, A.J.J., et al. Upper Extremity Function after Transradial Percutaneous Coronary Intervention: Short-Term Interim Results of the ARCUS Study. (2017) J Heart Cardiol 3(2): 34-39.

DOI: $10.15436 / 2378-6914.17 .1540$

Keywords: Coronary angioplasty; Percutaneous coronary intervention; Transradial artery access; Upper extremity function; Radial artery occlusion

\footnotetext{
Abbreviations

BCTQ: Boston Carpal Tunnel Questionnaire; DASH: Disabilities of the Arm, Shoulder and Hand; PCI: Percutaneous Coronary Intervention; SD: Standard deviation; UED: Upper Extremity Dysfunction; VAS: Visual Analogue Scale
}

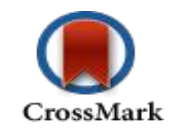




\section{Introduction}

The transradial access is being increasingly adopted as the preferred approach for angioplasties, as it is associated with significantly lower rates of procedural complications, shorter hospitalisations, greater patient satisfaction and lower costs than the transfemoral access, which, on the other hand, is technically less challenging and associated with a steeper learning curve ${ }^{[1-5]}$. However, the precise effects of transradial procedures on the upper extremities function are unknown. The few published studies lack clear definitions of outcomes and systematic protocols and report inconsistent relationships between access-site complications and upper extremity function ${ }^{[6-9]}$.

Several important factors combined contribute to the normal function of the upper extremities, including intact anatomy, blood supply and lymphatic drainage, muscle strength, range of motion, coordination and sensibility. The absence of pain is a prerequisite for a normal upper extremity function, since pain has negative repercussions on these other factors. Upper extremity function has been defined as "The physiological capacity in which the patient can use an anatomically intact upper limb in everyday activities" ${ }^{\text {[9] }}$. Procedural complications may be the source of Upper Extremity Dysfunction (UED).

We undertook this prospective, observational study, to examine the effects of transradial Percutaneous Coronary Interventions (PCI) on the function of the upper extremities, and to optimize the transradial access-technique.

\section{Methods}

The aims and design of the "Effects of trAnsRadial perCUtaneouS (ARCUS) coronary intervention on upper extremity function" study have been published previously ${ }^{[4]}$. A target enrolment of 500 patients has been planned.

\section{Diagnostic procedures}

Doppler ultrasound examinations (SD2 Doppler, Huntleigh) were performed to examine both radial arteries before and after the PCI. Pain, disability of the upper extremities and the presence of carpal tunnel syndrome were ascertained, using self-administered questionnaires, including, respectively, the Visual Analogue Scale (VAS) for pain, the Disabilities of the Hand, Arm and Shoulder (DASH) questionnaire, and the Boston Carpal Tunnel Questionnaire (BCTQ). Swelling of the hands and forearms was measured by volumetry, using an ordinary tape-measure, and sensibility of the fingertips was measured with the Weinstein Enhanced Sensory Test (WEST filaments, Connecticut Bio instruments). We measured the key and palmar grip to ascertain the strength of the hands and thumbs. Additionally, we measured the isometric strength of flexion and extension of the hands and wrists. All measurements were performed on both upper extremities, according to the clinical assessment recommendations of the American Society of Hand Therapists ${ }^{[10]}$. All measurements were made pre procedural, at 24 hours, at 2 weeks, and at 1 and 6 months after the index procedure.

\section{Percutaneous coronary intervention}

The operators were strongly advised to adhere to the international professional practice guidelines for the performance of $\mathrm{PCI}^{[11]}$. However, procedural preferences were left to the dis- cretion of each operator. After local anaesthesia of the wrist with a subcutaneous injection of $2 \%$ lidocaine, the radial artery was punctured with an introducer needle (Terumo Medical Corporation, Tokyo, Japan) using a modified Seldinger technique ${ }^{[12,13]}$. After introduction of the wire, the introducer needle was removed and a $6 \mathrm{~F}$ introducer sheath (Terumo) was inserted ${ }^{[9]}$. Heparin, 100IU/KG and a mixture of verapamil, $5 \mathrm{mg}$, nitroglycerin, $200 \mu \mathrm{g}$, and $10 \mathrm{ml}$ of saline solution were administered. A $6 \mathrm{~F}$ hydrophilic guiding catheter (PendraCare, Leek, and the Netherlands) was inserted through the sheath and balloon angioplasty or stent implantation was performed in $\geq 1$ coronary arteries. After completion of the procedure, the access-site was compressed using a transradial Band compression device (Terumo) injected with $13 \mathrm{cc}$ air. After two hours the compression device was gradually deflated in 24 hours, according to the manufacturer's protocol. The patient was provided with a sling and in case of swelling or haematoma of the arm with a minimal elastic compression bandage.

\section{Study endpoints}

The primary study endpoint is upper extremity function at two weeks compared to the pre procedural baseline measurements. Because of the absence of a precise, published definition and measurements of upper extremity function, hand experts tried to capture upper extremity function with the help of several examinations, performed according to the American Society of Hand Therapists ${ }^{[10]}$. The primary endpoint they composed is a binary score, absence or presence, of Upper Extremity Dysfunction (UED). Presence of UED is defined as a) a $\geq 1$-point increase in the score of the BCTQ, or b) presence of $\geq 2$ of the following criteria: 1) an increase in one of the questionnaire scores, 2) absence of radial artery signal on Doppler ultrasound examination, 3) $\geq 2 \mathrm{~cm}$ increase in forearm or hand volume, 4) a $\geq 1$ filament decrease in fingertips sensibility, and 5) a decrease in wrist, elbow, key or palmar grip strength (Table 1) ${ }^{[11]}$. Secondary endpoints that are discussed in this paper are the complications following transradial PCI and the referral to a hand surgeon or rehabilitation specialist.

Table 1: Composed primary endpoint; positive binary score* of upper extremity dysfunction.

$\geq 1$ point increase in either the symptom-severity score or the functional-status score of the BCTQ

$\geq 15 \%$ increase in the DASH compared to baseline

Increase in the VAS score regarding the upper extremity of $\geq 2$ points compared to baseline.

Absent signal of the radial artery during Doppler ultrasound examination.

Strength: Decrease in flexion and extension of the elbow and wrist compared to baseline.

$-\geq 60 \mathrm{~N}$ decrease in palmar grip strength compared to baseline.

$-\geq 12 \mathrm{~N}$ decrease in key grip strength compared to baseline.

$-\geq 15 \%$ decrease in isometric strength compared to baseline.

$\geq 1$ filament increase in sensibility of the hand according to the WEST, compared to baseline

$\geq 1 \mathrm{~cm}$ increase at volumetry of the hand, using the figure-of-eight method, compared to baseline.

$\geq 1 \mathrm{~cm}$ increase at volumetry of the forearm, measured circumferentially $8 \mathrm{~cm}$ distal of the medial epicondyle, compared to baseline. 
* Positive score (dysfunction present): $\geq 1$ point increase in BCTQ or $\geq 2$ criteria as specified above at two weeks follow-up

BCTQ: Boston Carpal Tunnel Questionnaire, DASH: Disabilities of Arm, Hand and Shoulder, VAS: Visual Analogue Scale, WEST: Weinstein Enhanced Sensory Test.

If there are clinical complaints present at follow-up, patients will be referred to a hand rehabilitation specialist. Subsequently, diagnosis and diagnostic procedures performed at the hand centre will be registered. Also, the administered therapy and upper extremity related absence of work in days will be registered ${ }^{[4]}$. These results will be published and analysed in the final paper.

\section{Statistical Analyses}

The details of the statistical analyses have been published previously ${ }^{[4]}$. The results are presented as means \pm SD and counts and percentages. Two groups of patients are distinguished; patients with or without upper extremity dysfunction according to protocol definitions. Between-group differences of dichotomous variables was analysed using Fisher's exact test. Categorical variables with more than two categories are analysed with the Pearson chi-square test. For normally distributed variables the independent sample t-test was used, and the Mann-Whitney $U$ test for continuous variables that are not normally distributed. Additionally, differences in the two upper extremity scores (intervention side - non-intervention side) collected in each patient will be analysed using Mc Nemar's test ${ }^{[4]}$. All tests were two-sided, with a statistical significance level set at $5 \%$.

\section{Interim results}

Baseline characteristics: An interim analysis with 200 of the envisioned 500 patients was pre specified in the study protocol. Of these 200 patients 191 patients completed the pre procedural baseline and 2 week follow-up examinations of the upper extremity function in the context of the ARCUS study. The other 9 patients were either lost to follow-up or missed the 2 week follow-up visit. The baseline characteristics of the entire sample and of the groups with and without UED are shown in Table 2.

Table 2: Baseline characteristics of the entire study sample and of the groups with and without upper extremity dysfunction.

\begin{tabular}{|l|c|c|c|}
\hline \multirow{2}{*}{ Men } & All patients & \multicolumn{2}{|c|}{ Upper extremity dysfunction } \\
\cline { 3 - 4 } & & Present n= 120 & Absent n= 71 \\
\hline Age, y & $155(81.2)$ & $95(79.2)$ & $60(84.5)$ \\
\hline Body mass index & $28.0 \pm 4.5$ & $28.1 \pm 4.7$ & $27.8 \pm 4.3$ \\
\hline Height & $176 \pm 9$ & $176 \pm 9$ & $177 \pm 9$ \\
\hline Smoking & \multicolumn{3}{|l}{} \\
\hline Current & $32(16.7)$ & $21(17.5)$ & $11(15.5)$ \\
\hline Previous & $94(49.2)$ & $53(44.2)$ & $41(57.7)$ \\
\hline Never & $63(32.9)$ & $45(37.5)$ & $18(25.4)$ \\
\hline Hypertension & $102(53.4)$ & $61(50.8)$ & $41(57.7)$ \\
\hline Dyslipidaemia & $63(32.9)$ & $36(30.0)$ & $27(38.0)$ \\
\hline Diabetes & $35(18.3)$ & $19(15.3)$ & $16(23.9)$ \\
\hline
\end{tabular}

\begin{tabular}{|l|c|c|c|}
\hline $\begin{array}{l}\text { Family history } \\
\text { of heart disease }\end{array}$ & $79(41.3)$ & $60(50.0)$ & $19(26.8)^{*}$ \\
\hline $\begin{array}{l}\text { Pre-existent dis- } \\
\text { ease of interven- } \\
\text { tion hand }\end{array}$ & $81(42.4)$ & $49(40.8)$ & $32(45.1)$ \\
\hline $\begin{array}{l}\text { Previous tran- } \\
\text { sradial percuta- } \\
\text { neous coronary } \\
\text { interventions }\end{array}$ & $63(32.9)$ & $38(31.7)$ & $25(35.2)$ \\
\hline
\end{tabular}

Values are means \pm SD or numbers $(\%)$ of observations; $* \mathrm{p}=0.002$; all other between-groups differences are statistically non-significant

\section{Group with upper extremity dysfunction}

According to our binary score 120 of the 191 patients $(62.8 \%)$ presented with dysfunction of the intervention hand at 2 weeks after the procedure. Figure 1 illustrates the various functions that were impaired. The main components of UED were a decrease in sensibility, observed in $41.7 \%$ of patients, $\mathrm{a} \geq 15 \%$ increase in the DASH questionnaire score in $38.3 \%$ of patients, and an increase in hand volume in $38.3 \%$ of patients.

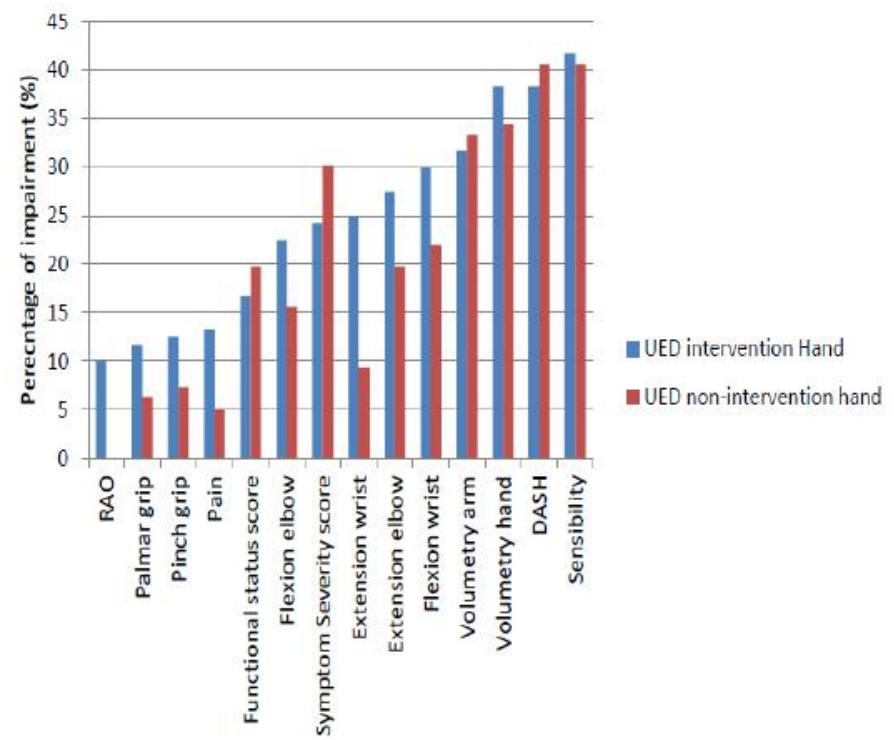

Figure 1: UED measurements in the intervention versus the non-intervention hand groups.

The measurements were made at 2 weeks compared with baseline in 120 patients with dysfunction of the intervention hand and in 96 patients with dysfunction of the non-intervention hand. RAO $=$ radial artery occlusion.

In absence of femoral control group, we analysed the same measurements in the non-intervention hands, which revealed dysfunction in 96 patients $(50.3 \%)$. In this group the main components of UED were a decrease in sensibility observed in $40.6 \%$ of patients, $\mathrm{a} \geq 15 \%$ increase in the DASH questionnaire score in $40.6 \%$ of patients, and an increase in volume of the hand in $34.4 \%$ of patients (Figure 1). There is a statistically significant difference $(\mathrm{p}<0.001)$ between UED in the intervention hand compared with the UED in the non-intervention hand.

\section{Complications}

At two weeks, the radial artery was occluded in 12 patients $(10 \%)$ in the UED group versus 1 patient $(1.4 \%)$ in the group without UED $(p=0.003$; table 3$)$. By Doppler ultrasound 
Transradial Percutaneous Coronary Intervention and upper extremity function

examination, the occlusion was total in two patients in the UED group and in the single patient without UED. In these patients presenting with radial artery occlusions, the main components of UED were an increase in all the questionnaire scores, a decrease in sensibility and a decrease in strength of extension of the wrist.

Table 3: Complications of transradial percutaneous coronary intervention.

\begin{tabular}{|l|c|c|}
\hline \multirow{2}{*}{} & \multicolumn{2}{|c|}{ Upper extremity dysfunction } \\
\cline { 2 - 3 } & Present $\mathbf{n}=\mathbf{1 2 0}$ & Absent $\mathbf{n}=\mathbf{7 1}$ \\
\hline Minor bleeding at access site & 0 & $1(1.4)$ \\
\hline Radial artery occlusion & $12(10.0)$ & $1(1.4)^{*}$ \\
\hline Access-site haematoma & $9(7.5)$ & $5(7.0)$ \\
\hline$>\mathbf{5} \mathbf{~ c m}$ & $14(11.6)$ & $4(5.6)$ \\
\hline$<\mathbf{5} \mathbf{~ c m}$ & $7(5.8)$ & $1(1.4)$ \\
\hline Swelling & $8(6.6)$ & $1(1.4)$ \\
\hline $\begin{array}{l}\text { Referral to hand rehabilita- } \\
\text { tion specialist after 2 weeks }\end{array}$ &
\end{tabular}

Values are means $\pm \mathrm{SD}$ or numbers ( $\%$ ) of observations; $* \mathrm{p}=0.003$; all other between-groups differences are statistically non-significant

\section{Clinical relevance}

After two weeks of follow-up, 8 patients with UED (6.6 $\%$ ) were referred for rehabilitation. The main components of UED in these patients were an increase in pain with $\geq 2$ points on the Visual Analogue Scale, an increase in DASH questionnaire and BCTQ scores, and a decrease in sensibility. The main complaint, which predominantly determined the patient referrals, was pain, often accompanied by a haematoma. The patients in the UED group referred for rehabilitation had an average of 5.7 \pm 2.3 points, therefore, nearly 6 positive criteria.

Patients with UED were significantly more likely to present with a family history of heart disease (50\% vs. $26.8 \%$ $; p=0.002)$.In addition, there was a trend $(p=0.07)$ toward a greater proportion of previous smokers in the UED group. In the group without dysfunction of the intervention hand, an asymptomatic music teacher was the only patient referred for rehabilitation and close surveillance after prolonged bleeding and development of a major haematoma at the access-site.

\section{Modified upper extremity dysfunction}

Our primary study endpoint emphasises the questionnaire scores, the BCTQ score in particular. However, these questionnaire scores are not side-specific and overestimate the dysfunction of the non-intervention hand. Furthermore, some cut-off points may be overly stringent. Therefore, we adjusted other criteria. We used absolute numbers initially to compare the strength, though percentages are more appropriate. A 60 Newton decrease for a young man is a minimal difference, whereas it is a large difference for an old lady. To increase the specificity, we have adjusted the cut-off points of several criteria (Table 4). These adjustments ensure that patients with positive scores present with clinically relevant UED. Table 4 further clarifies the modified primary endpoint. With these modified criteria, UED was present in the intervention hand in 33 patients $(16.9 \%)$, versus $6.2 \%$ in the non-intervention hand, probably approaching the true UED rate after transradial PCI.

Table 4: Modified upper extremity dysfunction.

\begin{tabular}{|c|c|c|c|c|}
\hline \multirow[b]{2}{*}{$\begin{array}{l}\text { Positive } \\
\text { scores for }\end{array}$} & \multicolumn{2}{|l|}{ Hand } & \multicolumn{2}{|c|}{ Statistical test } \\
\hline & $\begin{array}{l}\text { Interven- } \\
\text { tion }\end{array}$ & $\begin{array}{l}\text { Non-inter- } \\
\text { vention }\end{array}$ & McNemar & $\begin{array}{l}\text { Wilcoxon } \\
\text { signed } \\
\text { rank }\end{array}$ \\
\hline $\begin{array}{l}\text { Modified up- } \\
\text { per extremity } \\
\text { dysfunction }\end{array}$ & $33(16.8 \%)$ & $12(6.2 \%)$ & $<0.001^{*}$ & $<0.001^{*}$ \\
\hline $\begin{array}{l}\geq 2 \text { points } \\
\text { on visual } \\
\text { analogue pain } \\
\text { scale }\end{array}$ & $20(10.3 \%)$ & $7(3.6 \%)$ & $0.011 *$ & $0.007^{*}$ \\
\hline $\begin{array}{l}\text { Absent or } \\
\text { high pitch } \\
\text { Doppler signal }\end{array}$ & $13(6.7 \%)$ & $0(0 \%)$ & $<0.001^{*}$ & $<0.001 *$ \\
\hline \multicolumn{5}{|c|}{$\geq 2 \mathrm{~cm}$ increase in volume of the } \\
\hline Hand & $14(7.2 \%)$ & $11(5.6 \%)$ & 0.64 & 0.49 \\
\hline Forearm & $10(5.1 \%)$ & $11(5.6 \%)$ & 1.00 & 0.82 \\
\hline $\begin{array}{l}\geq 2 \text { filaments } \\
\text { decrease in } \\
\text { sensitivity }\end{array}$ & $32(16.4 \%)$ & $28(14.4 \%)$ & 0.70 & 0.56 \\
\hline \multicolumn{5}{|c|}{$<15 \%$ decrease in } \\
\hline $\begin{array}{l}\text { Key pinch } \\
\text { strength }\end{array}$ & $19(9.7 \%)$ & $13(6.7 \%)$ & 0.30 & 0.19 \\
\hline $\begin{array}{l}\text { Palmar grip } \\
\text { strength }\end{array}$ & $14(7.2 \%)$ & $7(3.6 \%)$ & 0.42 & 0.28 \\
\hline $\begin{array}{l}\text { Flexion of the } \\
\text { wrist }\end{array}$ & $38(19.5 \%)$ & $24(12.3 \%)$ & 0.80 & 0.61 \\
\hline $\begin{array}{l}\text { Extension of } \\
\text { the wrist }\end{array}$ & $30(15.4 \%)$ & $9(4.6 \%)$ & $0.039 *$ & $0.02 *$ \\
\hline $\begin{array}{l}\text { Flexion of the } \\
\text { elbow }\end{array}$ & $29(14.9 \%)$ & $17(8.7 \%)$ & 0.45 & 0.31 \\
\hline $\begin{array}{l}\text { Extension of } \\
\text { the elbow }\end{array}$ & $36(18.5 \%)$ & $21(10.8 \%)$ & $1.00 *$ & 0.80 \\
\hline
\end{tabular}

Positive UED score (dysfunction present): $\geq 2$ criteria as specified below at the two-week follow-up

$-\geq 2$ points increase in the Visual Analogue Scale score in the upper extremity

- Absent signal of the radial artery during Doppler ultrasound examination

$\bullet \geq 2 \mathrm{~cm}$ increase in hand volume, using the figure-of-eight method

- $\geq 2 \mathrm{~cm}$ increase in forearm volume, measured circumferentially, $8 \mathrm{~cm}$ distal of the medial epicondyle

$-\geq 2$ filaments increase in sensitivity of the hand by the Weinstein Enhanced Sensory Test

- Strength:

- $\geq 15 \%$ decrease in palmar grip strength compared to baseline.

$-\geq 15 \%$ decrease in key grip strength compared to baseline.

$\cdot \geq 15 \%$ decrease in isometric strength (flexion \& extension) of the wrist and elbow

\section{Discussion}

This pre specified interim analysis was conducted because this field of research is virtually a clean slate. There has never been such an extended and all-round study grasping all facets forming upper extremity function after transradial PCI. The highly sensitive binary score, composed by hand specialists, is a first attempt to measure the overall function of the upper extremity after transradial $\mathrm{PCI}^{[9]}$. We think that it may have been 
too sensitive and overestimated the actual and clinically relevant upper extremity dysfunction. We tentatively hypothesise that we chose unforgiving cut-off values, which lead to high false positive UED scores in both hands. Therefore the primary endpoint should be modified, to optimize the specificity.

Other potential causes of a high UED score, either in the intervention arm or the non-intervention arm, might have been a pre-existent disease of the hands, such as arthrosis, carpal tunnel syndrome or tendinitis. In patients presenting with upper extremity diagnoses, the pinch strength of the asymptomatic side may decrease from compensating which results in overuse and fatigue. This must be taken into account when interpreting pinch strength of the asymptomatic extremity ${ }^{[14]}$. Furthermore, by increasing the peripheral arterial osmolality and causing vasodilation $^{[15]}$, the contrast material may cause bilateral dysfunction. It may also injure the microvasculature and vascular endothelium by inducing apoptosis, and impair the physiological integrity resulting in UED, by altering vascular haemostasis, vessel growth, angiogenesis and permeability and tone of the blood vessel wall ${ }^{[16,17]}$. These disturbances may be observed with beta-adrenergic blockers and with statins, in the form of myalgia, myositis, myopathy, cramps and muscle weakness ${ }^{[18-20]}$. Diabetes may condition the prognosis of acute coronary syndromes and when regulated tight during the procedure it up-regulates the endothelial progenitor cell level and differentiation in patients going through an acute ST-elevation myocardial infarction ${ }^{[21,22]}$. We did not find this in our study.

Patients suffering from heart disease are hypoactive in the post procedural period, which might influence the questionnaire scores, and overestimate the degree of dysfunction. Furthermore, all questionnaire scores are not side-specific and could therefore overestimate the dysfunction on the intervention hand and non-intervention hand. We believe that in 39 patients $(20 \%)$, the dysfunction in the non-intervention hand could be explained by an unselective DASH score. Another $15 \%$ could be clarified by using absolute values of strength instead of percentages. Our presumption is that in another $10-15 \%$ it was induced by drugs.

The actual clinical relevance of our UED-score remains to be determined. We believe that the actual UED rate most likely will range between the rate of referrals for rehabilitation $(6.6 \%)$ and the modified UED rate $(16.9 \%)$. At the moment, the referral rate reflects the number of patients who had complaints and were urging for a referral to the hand rehabilitation specialist. However, during follow-up, several patients were candidates for referrals, though they were not all willing to see an additional physician and undergo further ambulatory clinic visits. They preferred to endure their disability, which caused an underestimation of the indications for referrals.

Keeping the complications and possible impairments in mind, custom treatments should be planned, according to professional and daily activities. Dialogs with the patients, for instance musicians, surgeons or interventional cardiologists are important, since the digital sensibility is one of the most often impaired functions, which could lower their professional performance.

In response to the results of this interim analysis, we already initiated a femoral control group. With this group, drug-induced complications could be demonstrated or excluded. Furthermore, the effects of pre-existent hand disease could be detected. Thus far, the non-intervention arm has been used as control. However, it is not accurate enough with respect to the questionnaires, since one needs both arms to open a bottle or to get dressed. Moreover, it would be worthwhile to focus on the prevention of UED by using slender and sheath less techniques, 4 or $5 \mathrm{~F}$ catheters and miniaturization of the PCI equipment, and more efficient use of the closure devices, for example using oximetry-guided patent haemostasis ${ }^{[23,24]}$. Furthermore, the OPERA trial, which focuses on prevention and treatment, is being launched. Imaging of the radial lumen, using optical coherence tomography will provide more insight into the injury caused to the vessel by transradial PCI and into the pathogenesis of radial artery occlusion, thus in greater expertise of prevention and treatment of complications. Additionally, a study regarding treatment of complications, cost effectiveness and early referral is on-going.

\section{Limitations of our study}

The results of this interim analysis should be interpreted cautiously. At the completion of our enrolment, multiple variable analyses will be performed, presumably yielding more reliable results. Furthermore, this study was not randomised; the measurements were made in both upper extremities and the non-intervention extremity was used as the control arm. To eliminate the confusion caused by the high rate of dysfunction observed in the non-intervention hand, a femoral control group has been introduced. This should isolate the true effect of transradial PCI on the upper extremity function by eliminating the possible effects of drug-induced myopathies. It should also be stressed that these are short-term, interim results, while the long-term results are awaited to reveal the true dimensions and scope of these findings.

\section{Conclusion}

UED after transradial PCI is a true medical concern. However, our original score may be overly sensitive and overestimates the rate of UED. Further analyses are needed, as well as perhaps a modification of the primary endpoint. We expect the true clinical relevance to be between $6.6 \%-16.9 \%$.

Funding: This work was supported by Terumo Europe with a grant for the building of a database. Dr. Zwaan was supported by a grant from the Carplast foundation, The Netherlands.

Conflict of Interest: The authors have no financial relationships or conflicts of interest to disclose regarding the contents of this article.

\section{References}


1. Caputo, R.P., Tremmel, J.A., Rao, S., et al. Transradial arterial access for coronary and peripheral procedures: Executive summary by the Transradial Committee of the SCAI. (2011) Catheter Cardiovasc Interv 78(6): 823-839.

Pubmed | Crossref | Others

2. Bertrand, O.F., Belisle, P., Joyal, D., et al. Comparison of transradial and femoral approaches for percutaneous coronary interventions: A systematic review and hierarchical Bayesian meta-analysis. (2012) Am Heart J 163(4): 632-648.

Pubmed | Crossref | Others

3. Jolly, S.S., Yusuf, S., Cairns, J., et al. Radial versus femoral access for coronary angiography and intervention in patients with acute coronary syndromes (RIVAL): A randomised, parallel group, multicentre trial. (2011) Lancet 377(9775): 1409-1420.

Pubmed | Crossref | Others

4. Zwaan, E.M., IJsselmuiden, A.J., Van Rosmalen, J., et al. Rationale and design of the ARCUS: Effects of trAnsRadial perCUtaneouS coronary intervention on upper extremity function. (2016) Cathet Cardiovasc Intervent 88(7): 1036-1043.

Pubmed | Crossref | Others

5. Louvard, Y., Kumar, S., Lefevre, T. Percentage of transradial approach for interventional cardiology in the world and learning the technique. (2009) Ann Cardiol Angeiol 58(6): 327-332.

Pubmed | Crossref | Others

6. Bazemore, E., Mann, J.T. Problems and complications of the transradial approach for coronary interventions: A review. (2005) J Invasive Cardiol 17(3): 156-159.

Pubmed | Crossref $\mid$ Others

7. Kanei, Y., Kwan, T., Nakra, N.C., et al. Transradial cardiac catheterization: A review of access site complications. (2011) Catheter Cardiovasc Interv 78(6): 840-846.

Pubmed | Crossref | Others

8. Bhat, T., Teli, S., Bhat, H., et al. Access-site complications and their management during transradial cardiac catheterization. (2012) Expert Rev Cardiovasc Ther 10(5): 627-634.

Pubmed | Crossref | Others

9. Zwaan, E.M., Koopman, A.G., Holtzer, C.A., et al. Revealing the impact of local access-site complications and upper extremity dysfunction post transradial percutaneous coronary procedures. (2015) Neth Heart J 23(11): 514-524.

Pubmed | Crossref | Others

10. Mac Dermi, J., Solomon, G., Valdes, K. ASHT Clinical Assessment Recommendations 3rd Edition: Impairment-Based Conditions. (2015) American Society of Hand Therapists 1-80.

Pubmed | Crossref | Others

11. Smith, S.C., Dove, J.T., Jacobs, A.K., et al. ACC/AHA guidelines for percutaneous coronary intervention: executive summary and recommendations: a report of the American College of Cardiology/American Heart Association Task Force on Practice Guidelines (Committee to Revise the 1993 Guidelines for Percutaneous Transluminal Coronary Angioplasty). (2001) Circulation 103: 3019-3041.

Pubmed | Crossref | Others

12. Seldinger, S.I. Catheter replacement of the needle in percutaneous arteriography; a new technique. (1953) Acta Radiol 39(5): 368-376.

Pubmed | Crossref | Others

13. Pancholy, S.B., Sanghvi, K.A., Patel, T.M. Radial artery access technique evaluation trial: randomized comparison of Seldinger versus modified Seldinger technique for arterial access for transradial catheterization. (2012) Catheter Cardiovasc Interv 80(2): 288-291.

Pubmed | Crossref | Others

14. MacDermi, J., Solomon, G., Valdes, K. ASHT Clinical Assessment Recommendations $3^{\text {rd }}$ Edition: Impairment-Based Conditions. (2015) $3^{\text {rd }}$ edition. American Society of Hand Therapists 21-31.

Pubmed $\mid$ Crossref $\mid$ Others

15. Higgins, C.B. Contrast media in the cardiovascular system. In: Sovak M, ed. (1984) Radio contrast Agents Berlin: Springer-Verlag 73: 193-251.

Pubmed $\mid$ Crossref $\mid$ Others

16. Zhang, H., Holt, C.M., Malik, N., et al. Effects of radiographic contrast media on proliferation and apoptosis of human vascular endothelial cells. (2000) Br J Radiol 73(874): 1034-1041.

Pubmed | Crossref | Others

17. Aspelin, P., Stacul, F., Thomsen, H.S., et al. Effects of iodinated contrast media on blood and endothelium. (2006) Eur Radiol 16(5): 1041-1049.

Pubmed | Crossref | Others

18. Ganga, H.V., Slim, H.B., Thompson, P.D. A systematic review of statin-induced muscle problems in clinical trials. (2014) Am Heart J 168(1): 6-15.

Pubmed | Crossref | Others

19. Lee, A. Adverse Drug Reactions. 2nd edition. (2009) Great Britain: Pharmaceutical Press.

Pubmed | Crossref $\mid$ Others

20. Bannwarth, B. Drug-induced myopathies. (2002) Expert Opin Drug saf 1(1): 65-70.

Pubmed | Crossref | Others

21. Balestrieri, M.L., Rizzo, M.R., Barbieri, M., et al. Sirtuin 6 expression and inflammatory activity in diabetic atherosclerotic plaques: effects of incretin treatment. (2015) Diabetes 64(4): 1395-1406.

Pubmed | Crossref | Others

22. Marfella, R., Rizzo, M.R., Siniscalchi, M., et al. Peri-procedural tight glycemic control during early percutaneous coronary intervention up-regulates endothelial progenitor cell level and differentiation during acute ST-elevation myocardial infarction: effects on myocardial salvage. (2013) Int J Cardiol 168(4): 3954-3962.

Pubmed | Crossref | Others

23. Kiemeneij, F., Yoshimachi, F., Matsukage, T., et al. Focus on maximal miniaturization of transradial coronary access materials and techniques by the Slender Club Japan and Europe: an overview and classification. (2015) Euro Intervention 10(10): 1178-1186.

Pubmed | Crossref | Others

24. Amoroso, G.1., van Dullemen, A., Westgeest, P., et al. "Virtual" 3 Fr Transradial Coronary Stenting With the 5 Fr Meito Masamune Sheathless Guiding Catheter: Feasibility and Safety in an Outpatient Setting. (2016) J Invasive Cardiol 28(3): 109-114.

Pubmed | Crossref $\mid$ Others
Ommega Online Publishers

Journal Title: Journal of Heart and Cardiology

Short title : J Heart Cardiol
Journal ISSN: 2378-6914 (online)

Journal E-mail: cardiology@ommegaonline.com

Website: www.ommegaonline.org 